\title{
Least square application on frequency weighted model reduction technique
}

\begin{abstract}
In this paper, we present new results on frequency weighted balanced truncation technique based on vec and Kronecker Product. This technique is applied to single-sided case and an extension to double-sided case is also presented. Numerical example with comparisons to other techniques is also presented.
\end{abstract}

